\title{
Optimization and enrichment of induced cardiomyocytes derived from mouse fibroblasts by reprogramming with cardiac transcription factors
}

\author{
JIAXIN TIAN $^{1^{*}}$, RONG WANG ${ }^{2 *}$, QIAN HOU $^{3 *}$, MEIRONG LI $^{3}$, LI CHEN $^{3}$, XIANGDONG DENG $^{3}$, \\ ZIYING ZHU $^{3}$, YALI ZHAO ${ }^{3}$, WENJUN $\mathrm{HE}^{3,4}$ and XIAOBING FU $\mathrm{FU}^{3,4}$ \\ ${ }^{1}$ Centre for Medical Device Evaluation, China Food and Drug Administration, Beijing 100044; \\ ${ }^{2}$ Department of Cardiovascular Surgery, Institute of Cardiac Surgery of PLA; ${ }^{3}$ Institute of Basic Medicine, \\ Chinese PLA General Hospital, Beijing 100853; ${ }^{4}$ Wound Healing and Cell Biology Laboratory, \\ The First Affiliated Hospital of Chinese PLA General Hospital, Beijing 100048, P.R. China
}

Received May 5, 2017; Accepted September 29, 2017

DOI: $10.3892 / \mathrm{mmr} .2017 .8285$

\begin{abstract}
Ischemic heart disease within developed countries has been associated with high rates of morbidity and mortality. Cell-based cardiac repair is an emerging therapy for the treatment of cardiac diseases; however, a limited source of the optimal type of donor cell, such as an autologous cardiomyocyte, restricts clinical application. The novel therapeutic use of induced pluripotent stem cells (iPSCs) may serve as a unique and unlimited source of cardiomyocytes; however, iPSC contamination has been associated with teratoma formation following transplantation. The present study investigated whether cardiomyocytes from mouse fibroblasts may be reprogrammed in vitro with four cardiac transcription factors, including GATA binding protein 4 , myocyte-specific enhancer factor $2 \mathrm{C}$, T-box transcription factor 5, and heartand neural crest derivatives-expressed protein 2 (GMTH). Cardiac-specific markers, including $\alpha$-myosin heavy chain
\end{abstract}

Correspondence to: Dr Xiaobing Fu, Institute of Basic Medicine, Chinese PLA General Hospital, 28 Fuxing Road, Haidian, Beijing 100853, P.R. China

E-mail: fuxiaobing@vip.sina.com

Dr Wenjun He, Wound Healing and Cell Biology Laboratory, The First Affiliated Hospital of Chinese PLA General Hospital, 51 Fucheng Road, Haidian, Beijing 100048, P.R. China

E-mail: 520hewenjun@163.com

*Contributed equally

Abbreviations: iPSCs, induced pluripotent stem cells; GMTH, Gata4, Mef2c, Tbx5 and Hand2; GMT, Gata4, Mef2c and Tbx5; iCMs, induced cardiomyocytes; ESCs, embryonic stem cells; TTFs, tail-tip fibroblasts; CIM, cardiac inductive medium; SHF, second heart field; BMP, bone morphogenetic protein

Key words: direct reprogramming, enrichment, induced cardiomyocytes, fibroblasts, Percoll density centrifugation
( $\alpha$-MHC), $\beta$-MHC, atrial natriuretic factor, NK2 homeobox 5 and cardiac troponin $\mathrm{T}$ were observed within mouse fibroblasts reprogrammed with GMTH, which was reported to be more effective than GMT. In addition, Percoll density centrifugation enriched a population of $\sim 72.4 \pm 5.5 \% \alpha-\mathrm{MHC}^{+}$induced cardiomyocytes, which retained the expression profile of cardiomyocyte markers and were similar to natural neonatal cardiomyocytes in well-defined sarcomeric structures. The findings of the present study provided a potential solution to myocardial repair via a cell therapy applying tissue engineering with minimized risks of immune rejection and tumor formation.

\section{Introduction}

Ischemic heart disease within developed countries has been associated with high rates of morbidity and mortality (1). Heart failure following acute myocardial infarction may arise from a marked loss of cardiomyocytes due to the reduced regenerative ability of adult cardiomyocytes; however, a previous study reported that cell-based cardiac repair may serve as a novel treatment with promising results (2). A variety of cell types have previously been employed to investigate the underlying mechanism of heart tissue repair (3). The ideal cell type for transplantation constitutes a cardiomyocyte with natural electrophysiological, structural and contractile properties. Pluripotent stem cells (PSCs) as a source of cardiomyocytes may be considered as a unique and infinite source of cells for the development of regenerative medicine. Embryonic stem cells (ESCs) have been reported to produce bona fide cardiomyocytes (4,5); however, the use of ESCs in a clinical setting has been associated with tumor formation, cellular rejection and various ethical issues. To revert cardiomyocytes into the induced (i)PSC form, four transcription factors may be employed $(6,7)$ to produce a source of autologous SCs; the use of iPSCs may avoid the risk of immune rejection, however, teratoma formation may occur.

In contrast to pluripotent reprogramming, direct reprogramming of somatic cells, including easily accessible 
adipocytes and dermal fibroblasts into therapeutic cell types exhibiting tissue-specific transcription factor overexpression, may serve as a valuable source of cells suitable for direct clinical application (8-12). Brahma-associated factor 60c, a chromatin remodeling factor, in combination with GATA binding protein 4 (GATA4) and T-box transcription factor 5 (TBX5) was reported to induce the trans-differentiation of the mouse mesoderm intocardiac tissue (13). It has been reported that postnatal cardiac or dermal fibroblasts may be rapidly and efficiently reprogrammed into differentiated cardiomyocyte-like cells, with a combination of three cardiac-specific transcription factors, namely, GATA4, myocyte-specific enhancer factor 2C (MEF2C), and TBX5 (GMT) (9). Additionally, fibroblasts may exhibit a cardiomyocyte-like phenotype via a combination of microRNAs1, 133, 208 and 499, in vitro and in vivo (14). These strategies exclude the risk of teratoma formation as cells are not reverted to a pluripotent stage; lineage reprogramming may be used to convert cell fate in situ $(15,16)$. Progress towards cardiac reprogramming has been attained in previous studies $(17,18)$; however, the effects of induced cardiomyocytes (iCMs) remain to be investigated. Further investigation is required to identify unknown combinations of transcription factors that exhibit greater potency in cardiac reprogramming. Lineage reprogramming technology may convert fibroblasts into iCMs; however, the percentage of fully reprogrammed cardiomyocytes from fibroblasts was low in vitro. Applying these findings to clinical practice is dependent upon the improvement of cardiomyocyte enrichment, as contaminated cell types may markedly attenuate drug responses and other functional properties in vitro and in vivo (19).

The aims of the present study were to investigate the optimal combination of key cardiac transcription factors to effectively reprogram mouse fibroblasts into $\mathrm{iCMs}$ and to determine whether iCMs may be enriched via Percoll density centrifugation. The combination of the four transcription factors, GMT with heart- and neural crest derivatives-expressed protein 2 (HAND2; GMTH), was compared with the combination of GMT within mouse fibroblasts. iCMs reprogrammed from GMTH-transduced fibroblasts were successfully enriched via Percoll density centrifugation. Enriched iCMs constituted $\geq 72.4 \pm 5.5 \% \alpha-\mathrm{MHC}^{+}$cells, which retained the cardiac phenotype, and may serve as a valuable source of cells for the development of cardiovascular disease research and clinical treatment, with minimized risks of immune rejection and tumor formation.

\section{Materials and methods}

Plasmid construction. pWPT lentiviral vectors (plasmid cat. no. 12255; Addgene, Inc., Cambridge, MA, USA) were constructed using the coding regions of mouse GATA4, $M E F 2 C, T B X 5$ and $H A N D 2$ genes, which were amplified via reverse transcription-polymerase chain reaction (RT-PCR, RR014A; Takara Biotechnology Co., Ltd., Dalian, China). The primers sequences used are listed in Table I, and denaturation at $94^{\circ} \mathrm{C}$ for $30 \mathrm{sec}$, annealing at $60^{\circ} \mathrm{C}$ for $30 \mathrm{sec}$, extension at $72^{\circ} \mathrm{C}$ for $2 \mathrm{~min}$, and 35 cycles were used. The sequences were inserted into the BamHI/SalI or MluI/SalI site of the pWPT plasmid. A Kozak sequence, GCCACC, was inserted prior to the ATG codon; amplification of the target cDNA fragment was achieved using a 5'-primer containing a Kozak sequence and an ATG codon, and a 3'-primer with unique restriction sites. Primer sequences were introduced into the multiple cloning site of the pWPT lentiviral vector; a stop codon at the 3 '-end of the target sequence was omitted.

Cell culture. The tail-tip fibroblasts (TTFs) used in the present study were isolated from 10 male ICR mice (5 day-old). Briefly, mice were anesthetized with $2 \%$ diethyl ether for $15 \mathrm{sec}$, and tails were removed and then rinsed in ethanol followed by PBS. The tissue was minced, transferred into a solution constituting $0.25 \%$ trypsin, $0.2 \%$ EDTA and $0.1 \%$ collagenase (Roche Diagnostics, Basel, Switzerland), and incubated at $37^{\circ} \mathrm{C}$ for $1 \mathrm{~h}$. Following centrifugation at $500 \mathrm{x} g$ at room temperature for 5 min, isolated cells were resuspended in Dulbecco's modified Eagle's medium [DMEM; containing 10\% fetal bovine serum (FBS); Gibco; Thermo Fisher Scientific, Inc., Waltham, MA, USA] and seeded onto $100 \mathrm{~mm}$ dishes. TTFs of passages 2 to 3 were employed for cardiomyocyte induction. 293T cells (CRL-3216; American Type Culture Collection, Manassas, VA, USA), which were used to produce lentiviruses, were maintained in DMEM (containing 10\% FBS; Gibco; Thermo Fisher Scientific, Inc.). All procedures with experimental animals were approved by the Institutional Animal Care and Use Committee of Chinese PLA General Hospital (Beijing, China) and carried out according to the guidelines of China Council on Animal Care and Use (permit no. 2014-X9-09).

Lentivirus production. Prior to transduction, 293T cells were seeded at $8 \times 10^{6}$ cells $/ 100 \mathrm{~mm}$ dish. On the subsequent day, pWPT lentiviral vectors were transduced into $293 \mathrm{~T}$ cells using Lipofectamine ${ }^{\circledR} 2000$ (Invitrogen; Thermo Fisher Scientific, Inc.) transfection reagent according to the manufacturer's instructions. A total of $35 \mu \mathrm{l}$ transfection reagent, $8 \mu \mathrm{g}$ lentiviral expression plasmid, $4 \mu \mathrm{g}$ psPAX 2 and $2 \mu \mathrm{g}$ pMD2.G (all from Addgene, Inc.) were diluted in $3 \mathrm{ml}$ Opti-MEM ${ }^{\circledR}$ I medium (Thermo Fisher Scientific, Inc.), and then added dropwise onto 293T cells of $\sim 85 \%$ confluence; cells were maintained in $10 \mathrm{ml}$ fresh DMEM on $100 \mathrm{~mm}$ dishes. pWPT-GFP (Addgene, Inc.) was used as a negative control. At 48 and $72 \mathrm{~h}$ following transduction, the viral supernatants were collected, filtered using a $0.45 \mu \mathrm{m}$ pore size filter (EMD Millipore, Billerica, MA, USA) and concentrated 30-fold with PEG-it ${ }^{\mathrm{TM}}$ Virus Precipitation Solution (System Biosciences, Palo Alto, CA, USA). They were centrifuged at $1,500 \times \mathrm{g}$ at $4^{\circ} \mathrm{C}$ for $30 \mathrm{~min}$, and subsequently resuspended in PBS.

Preparation of $i C M s$. Mouse TTFs were seeded onto gelatin-coated $100 \mathrm{~mm}$ dishes at $1.5 \times 10^{4}$ cells $/ \mathrm{cm}^{2}$. Following initial cell culture, the culture medium was replaced with $10 \mathrm{ml}$ fresh DMEM containing 10\% FBS and $10 \mu \mathrm{g} / \mathrm{ml}$ polybrene (Sigma-Aldrich; Merck KGaA, Darmstadt, Germany)-this was classed as day 0 . Prior to overnight incubation at $37^{\circ} \mathrm{C}$, the same volume $(500 \mu \mathrm{l})$ of supernatant containing each of the GMTH lentiviruses were mixed and transferred to the dishes. In the control group, TTFs were untransduced or transduced with green fluorescent protein (GFP) lentivirus (Addgene, Inc.) On day 1, the medium of certain plates of GMTH-transduced TTFs was replaced with $10 \mathrm{ml}$ cardiac inductive medium (CIM) 
constituting DMEM/medium199 (4:1), 10\% FBS, 10\% conditioned medium obtained from neonatal mouse cardiomyocyte culture, $1 \%$ non-essential amino acids, vitamin mixture, 5\% horse serum, B-27, insulin-selenium-transferrin and sodium pyruvate (Invitrogen; Thermo Fisher Scientific, Inc.). GMTH-transduced cells were not treated with CIM as control. The medium was replaced every 2 days. Conditioned medium was filtered usinga $0.22 \mu \mathrm{m}$ pore size filter (EMD Millipore).

Percoll density centrifugation. TTFs $\left(1.5 \times 10^{6}\right)$ treated with CIM following GMTH transduction were dissociated, resuspended and loaded onto a discontinuous Percoll gradient on day 9. Percoll (GE Healthcare, Chicago, IL, USA) was diluted in a buffer solution containing $150 \mathrm{mM} \mathrm{NaCl}$ and $20 \mathrm{mM} \mathrm{4-(2-hydroxyethyl)-1-piperazineethanesulphonic}$ acid. The gradient consisted of a $40.5 \%$ Percoll layer above a layer of 58.5\% Percoll; cell layers were observed following centrifugation for $30 \mathrm{~min}$ at $1,500 \mathrm{xg}$ at room temperature. Cells of different layers were collected, washed with PBS and resuspended in DMEM medium. They were then plated for immunostaining, or collected for reverse transcription (RT)-qPCR analysis. The fractionated cells were seeded onto chamber slides, cultured for an additional few days and immunostained prior to immunocytochemical analysis.

$R T$-semi (s)-qPCR and $q P C R$. Total RNA was isolated from cultured cells (TTFs, TTFs + GMT and TTFs + GMTH) using TRIzol (Invitrogen; Thermo Fisher Scientific, Inc.) according to the manufacturer's instructions. For RT-sqPCR, cDNA was synthesized with RevertAid First Strand cDNA Synthesis kit (Thermo Fisher Scientific, Inc.) according to the manufacturer's instructions. PCR analyses were performed with $2 X$ Taq PCR MasterMix (Tiangen Biotech Co., Ltd., Beijing, China) according to the manufacturer's instructions. Thermocycling condition were: Denaturation at $94^{\circ} \mathrm{C}$ for $30 \mathrm{sec}$, annealing at $58^{\circ} \mathrm{C}$ for $30 \mathrm{sec}$, extension at $72^{\circ} \mathrm{C}$ for $30 \mathrm{sec}$, for 30 cycles. The products were run in $1 \%$ agarose gel with ethidium bromide, and images were captured with Gel $\mathrm{Doc}^{\mathrm{TM}} \mathrm{XR}+\mathrm{Gel}$ Documentation system (Bio-Rad Laboratories, Inc., Hercules, CA, USA). qPCR was performed with the Rotor-Gene ${ }^{\circledR} 2000$ system using the QuantiTect SYBR Green one-step RT-PCR kit and QuantiTect Primer Assay (both from Qiagen Co., Ltd., Shanghai, China) according to the manufacturer's instructions. The thermocycling conditions were as follows: $95^{\circ} \mathrm{C}$ for $15 \mathrm{sec}$, $60^{\circ} \mathrm{C}$ for $1 \mathrm{~min}$, for 40 cycles. The results were analyzed using the $2^{-\Delta \Delta \mathrm{Cq}}$ method (20) with GAPDH as a reference gene and GFP-transduced TTF cells as a calibrator. Primer sequences are presented in Table II.

Immunocytochemistry. Immunostaining was performed as previously described (21). Briefly, transduced and untransduced TTF cells were rinsed with PBS and fixed with 4\% paraformaldehyde for $15 \mathrm{~min}$ at room temperature. The fixed cells were washed with PBS three times and subsequently treated with $0.5 \%$ Triton X-100 for $30 \mathrm{~min}$ at room temperature. Unspecific binding sites were blocked by $5 \%$ bovine serum albumin (Sigma-Aldrich; Merck KGaA) for 30 min at room temperature. The primary antibodies against GATA4 (1:100, cat. no. sc-25310), MEF2C (1:100, cat. no. sc-13268), TBX5 (1:100, cat. no. sc-376952), HAND2 (1:100, cat.
Table I. Primer sequences for reverse transcription-polymerase chain reaction to amplify the candidate genes.

\begin{tabular}{lc}
\hline Genes & \multicolumn{1}{c}{ Sequence (5'-3') } \\
\hline GATA4 & F: GATCGGATCCGCCACCATGTACCAAAG \\
& CCTGGCCATGGC \\
& R: GATCGTCGACCGCGGTGATTATGTCCC \\
& CATGACT \\
MEF2C & F: GATCGGATCCGCCACCATGGGGAGAA \\
& AAAAGATTCAGATTACG \\
& R: GATCGTCGACTGTTGCCCATCCTTCAG \\
& AGAG \\
TBX5 & F: GATCGGATCCGCCACCATGGCCGATAC \\
& AGATGAGGG \\
& R: GATCGTCGACGCTATTCTCACTCCACT \\
& CTGG \\
HAND2 & F: GATCGGATCCGCCACCATGAGTCTGGT \\
& GGGGGGCTTTC \\
& R: GATCGTCGACCTGCTTGAGCTCCAGG \\
& GCCC
\end{tabular}

F, forward; R, reverse; Gata4, GATA binding protein 4; Hand2, heart- and neural crest derivatives-expressed protein 2; Mef2c, myocyte-specific enhancer factor 2C; Tbx5, T-box transcription factor 5 .

no. sc-9409), $\alpha$-myosin heavy chain (MHC, 1:100, cat. no. sc-20641) (all from Santa Cruz Biotechnology, Inc., Dallas, TX, USA), $\alpha$-sarcomeric actinin (1:100, cat. no. BM0003; Boster Biological Technology, Wuhan, China) and cardiac troponin T (cTnT, 1:200, cat. no. MAB-0374; Fuzhou Maixin Biotechnology, Fuzhou, China) were applied without washing and incubated at $4^{\circ} \mathrm{C}$ overnight. Following washing with PBS, cells were incubated with the secondary antibodies, fluorescein isothioyanate (FITC)-labeled or phalloidin-tetramethyl rhodamine-labeled goat anti-mouse immunoglobulin $\mathrm{G}$ ( $\mathrm{IgG}$, 1:50; OriGene Technologies, Inc., Rockville, MD, USA) in the dark at room temperature for $2 \mathrm{~h}$. Nuclei were stained with $0.1 \mu \mathrm{g} / \mathrm{ml}$ DAPI (Sigma-Aldrich; Merck KGaA) for $15 \mathrm{~min}$ at room temperature. Images were captured by an inverted phase contrast fluorescence microscopy (Olympus Corporation, Tokyo, Japan) and confocal laser scanning microscopy (Zeiss $\mathrm{GmbH}$, Jena, Germany).

Flow cytometric analysis. Cells were washed with PBS, dissociated with $0.25 \%$ trypsin and $0.04 \%$ EDTA, and then filtered through a $35 \mu \mathrm{m}$ cell-strainer to remove cell clumps. The resulting single cell suspensions were centrifuged at $300 \mathrm{x} \mathrm{g}$ for $5 \mathrm{~min}$ at room temperature, resuspended in $1 \mathrm{ml}$ PBS, pipetted into $1.5 \mathrm{ml}$ centrifuge tubes, further centrifuged at $400 \mathrm{xg}$ for $5 \mathrm{~min}$ at room temperature, and resuspended in $1 \mathrm{ml}$ 4\% paraformaldehyde at room temperature for $10 \mathrm{~min}$. Cells were collected by centrifugation at $400 \mathrm{x} \mathrm{g}$ for $5 \mathrm{~min}$ at room temperature and rinsed with PBS three times, and were then resuspended in $0.5 \%$ Triton X-100 for $30 \mathrm{~min}$ at room temperature. Subsequently, TTFs were stained with Anti-Myosin Heavy Chain Alexa Fluor ${ }^{\circledR} 488$ (1:100, cat. no. 53-6503-82; 
Table II. Primer sequences for semi-quantitative and quantitative polymerase chain reaction.

\begin{tabular}{lll}
\hline Gene & \multicolumn{1}{c}{ Forward sequence (5'-3') } & Backward sequence (5'-3') \\
\hline$\alpha-\mathrm{MHC}$ & ACCGTGGACTACAACAT & CTTTCGCTCGTTGGGA \\
$\beta-\mathrm{MHC}$ & ACCCCTACGATTATGCG & GTGACGTACTCGTTGCC \\
ANF & GGGGGTAGGATTGACAGGAT & CAGAGTGGGAGAGGCAAGAC \\
NKX2.5 & AGCAACTTCGTGAACTTTG & CCGGTCCTAGTGTGGA \\
CTNT & TGAGAGGAGGAAGGTGCTGG & CGCGGGTCTTGGAGACTTTC \\
GJA1 & CTTTCATTGGGGGAAAGGCG & AGCGAAAGGCAGACTGTTCA \\
GAPDH 1 2 & CACCCCAGCGAAGAACTCAT & TCTCCTCATCCAGGTACGCA \\
\hline
\end{tabular}

MHC, myosin heavy chain; ANF, natriuretic peptide A; NKX2.5, NK2 homeobox 5; CTNT, cardiac troponin T; GJA1, gap junction $\alpha 1$; COL1A2, collagen type 1 a2.
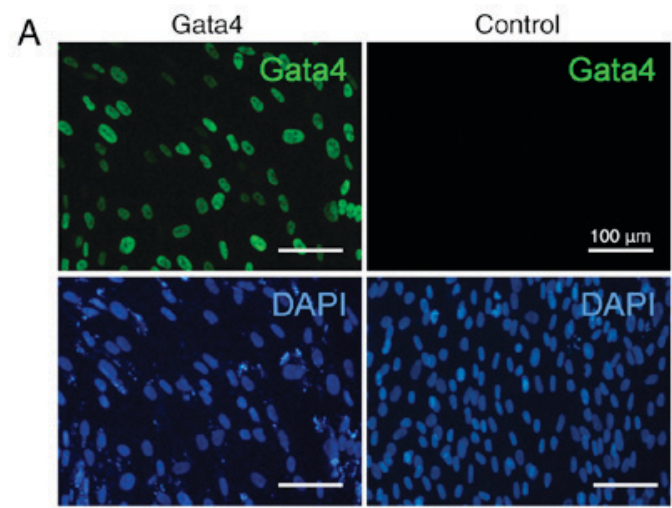

C
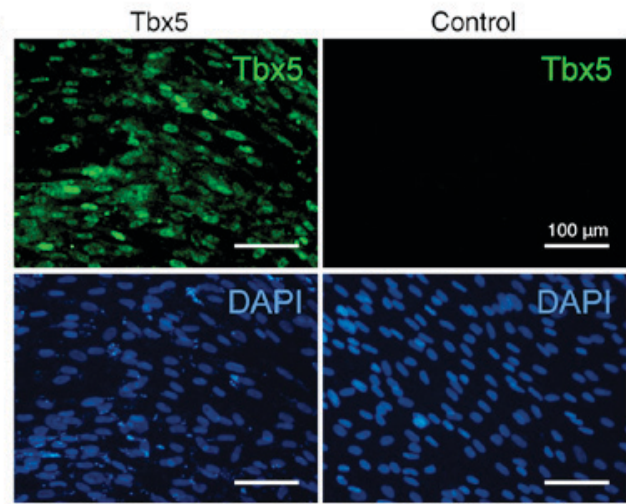

$\mathrm{D}$

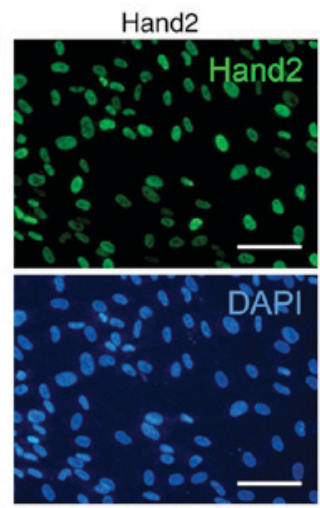

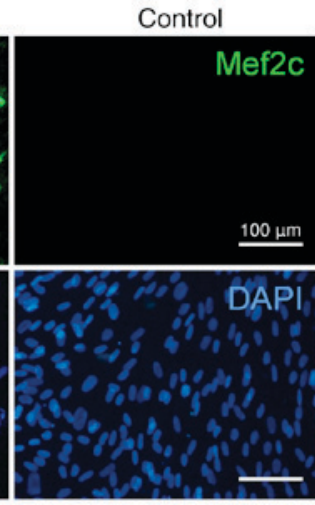

Figure 1. Candidate factors for cardiomyocyte induction. TTFs were transduced with individual lentiviruses expressing (A) GATA4 (B) MEF2C (C) TBX5 and (D) HAND2, and were then compared with untransduced TTFs, which were used as the control. Cells were immunostained with corresponding antibodies and DAPI. Scale bars, $100 \mu \mathrm{m}$. TTFs, tail-tip fibroblasts; GATA4, GATA binding protein 4; MEF2C, myocyte-specific enhancer factor 2C; TBX5, T-box transcription factor 5; HAND2, heart- and neural crest derivatives-expressed protein 2.

eBioscience; Thermo Fisher Scientific, Inc.) for $30 \mathrm{~min}$ at $37^{\circ} \mathrm{C}$, followed by three washes with PBS and centrifugation at $400 \mathrm{x} \mathrm{g}$ for $5 \mathrm{~min}$ at room temperature. The isotype control antibody was FITC-conjugated mouse IgG1; TTF pellets were resuspended in $500 \mu \mathrm{l}$ PBS to be detected with flow cytometer (FACSCalibur; BD Biosciences, Franklin Lakes, NJ, USA) for flow cytometric analysis (CellQuest Pro software version 5.1; BD Biosciences).

Statistical analyses. Results were repeated three times and expressed as the mean \pm standard deviation. Differences between groups were examined by Student's t-test for statistical significance using SPSS software version 4.0 (SPSS Inc., Chicago, IL, USA). $\mathrm{P}<0.05$ was considered to indicate a statistically significant difference.

\section{Results}

Generation of lentiviruses encoding individual cardiac transcription factors. The generated individual lentiviruses efficiently expressed each of the four cardiac-specific transcription factors GATA4, MEF2C, TBX5 and HAND2 


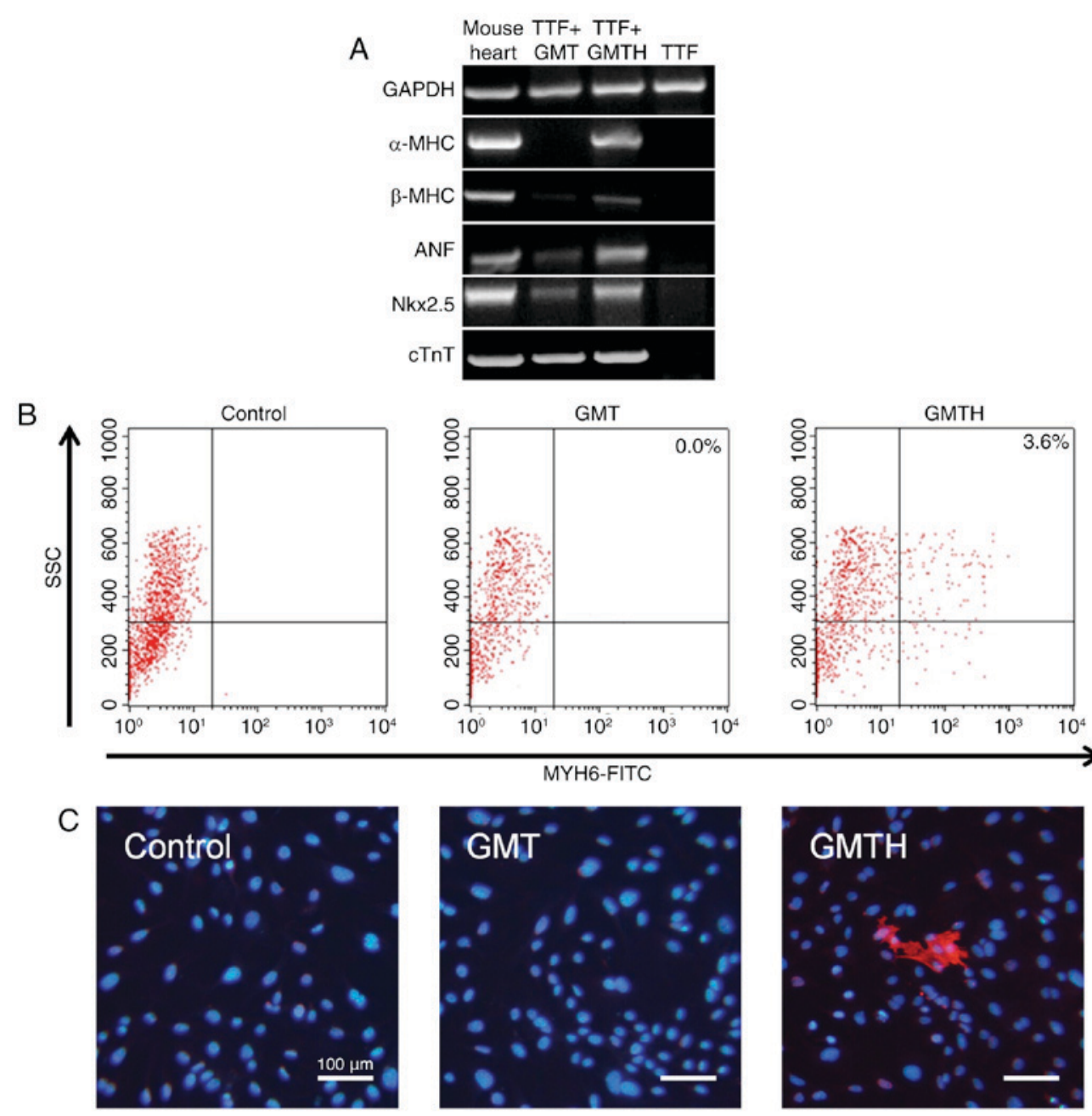

Figure 2. Reprogramming of TTFs into cardiac-like myocytes. (A) Semi-quantitative polymerase chain reaction analysis of cardiac-specific genes in mouse heart tissue, GMT-transduced TTFs, GMTH-transduced TTFs and the control TTFs two weeks post-transduction. (B) Flow cytometric analysis of $\alpha$-MHC expression in TTFs only or those transduced with GMT or GMTH. (C) Immunofluorescent staining of the control TTFs, GMT-infected and GMTH-infected TTFs for $\alpha$-MHC (red) and DAPI (blue). Scale bars, $100 \mu \mathrm{m}$. $\alpha$-MHC, $\alpha$-myosin heavy chain; GMT, GATA binding protein 4 , myocyte-specific enhancer factor $2 \mathrm{C}$ and T-box transcription factor 5; GMTH, GATA binding protein 4, myocyte-specific enhancer factor 2C, T-box transcription factor 5 and heart- and neural crest derivatives-expressed protein 2; TTFs, tail-tip fibroblasts; ANF, atrial natriuretic peptide; Nkx2.5, NK2 homeobox 5; cTnT, cardiac troponin T; SSC, side scattered light.

within mouse TTFs. Transduced or untransduced TTFs were examined on day 2 post-transduction, and the majority of transduced cells expressed cardiac transcription factors. Immunofluorescence staining for GMTH proteins demonstrated their nuclear localization (Fig. 1), and viral transduction efficiency was detected to be $>96 \%$.

Reprogramming of TTFs into cardiac-like myocytes. The effects of GMT were compared with those of GMTH in the present study. RT-PCR revealed that cardiac-specific genes were not expressed within the control TTFs compared with the GMT- and GMTH-transduced TTFs. The expression of some cardiac-specific genes within the GMT-transduced TTFs were detected; however, $\alpha$-MHC expression was not detected 14 days post-transduction. Conversely, GMTH-transduced TTFs demonstrated increased expression levels of cardiomyocyte markers, including $\alpha$-MHC, $\beta$-MHC, atrial natriuretic factor, NK2 homeobox $5(\mathrm{Nkx} 2.5)$ and cTnT compared with GMT transduction (Fig. 2A). Flow cytometry analysis confirmed that $\sim 3.6 \%$ of GMTH-transduced TTFs were positive for $\alpha$-MHC expression; however, $\alpha$-MHC ${ }^{+}$TTFs were not detected two weeks following GMT-transduction (Fig. 2B). Additionally, immunofluorescence staining indicated that some GMTH-transduced TTFs expressed $\alpha$-MHC, compared with in the control and GMT-transduced groups, in which no positive cells were detected (Fig. 2C).

Optimization of cardiac reprogramming by GMTH induction. To enhance the productivity of $\alpha-\mathrm{MHC}^{+}$cells, CIM was employed. CIM treatment stimulated the expression of $\alpha$-MHC within GMTH-transduced TTFs by 3 -fold, when compared with in untreated GMTH-transduced TTFs (Fig. 3A); no effect was detected in control TTFs. The duration of cardiomyocyte induction from TTFs was analyzed by flow cytometry. $\alpha-\mathrm{MHC}^{+}$cells were detected as early as 3 days following GMTH induction via CIM treatment, the number of $\alpha-\mathrm{MHC}^{+}$cells slowly increased to $\leq 18 \%$ at day 9 (Fig. 3B). The percentage of $\alpha-\mathrm{MHC}^{+}$cells peaked at day 9 post-transduction, and declined thereafter due to overgrowth of non-reprogrammed fibroblasts (Fig. 3C). 

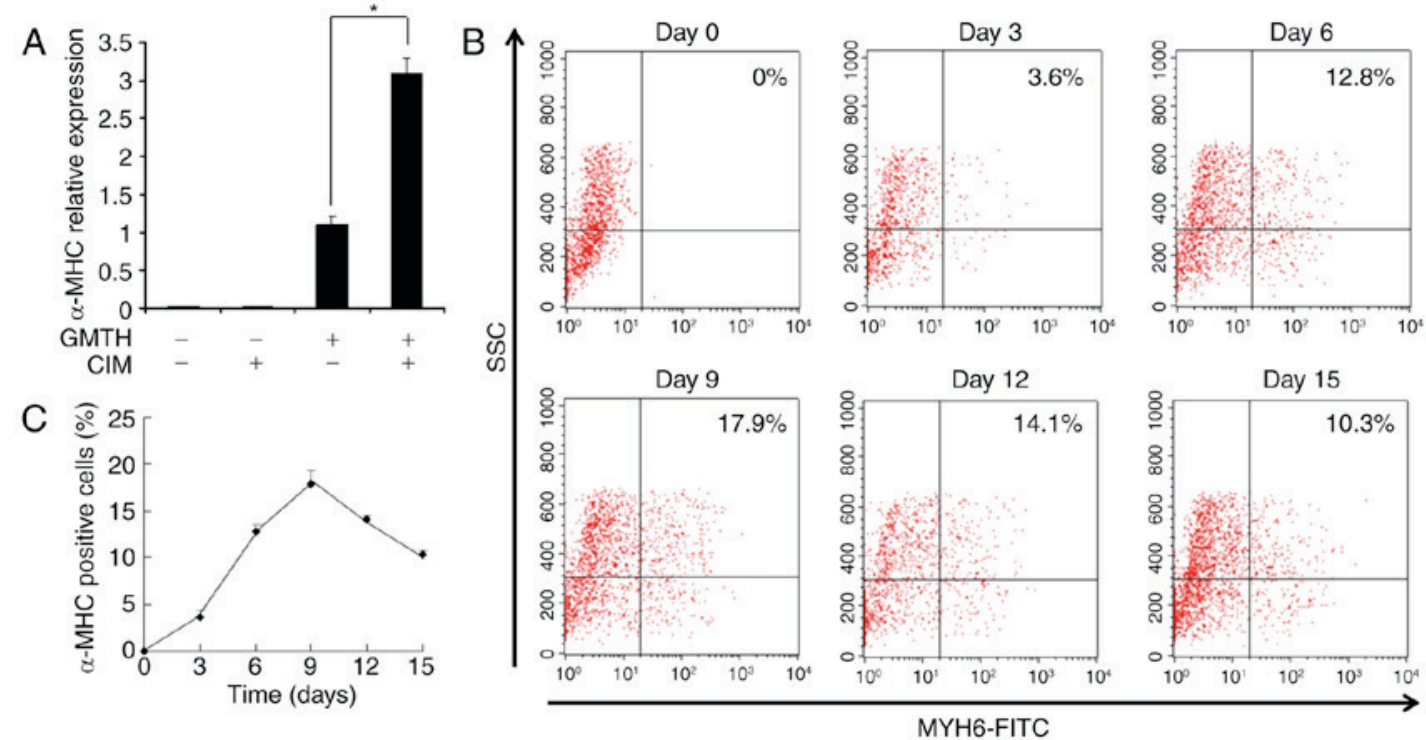

Figure 3. Optimization of cardiac reprogramming. (A) Quantitative polymerase chain reaction analysis of the $\alpha$-MHC gene in TTFs with or without CIM treatment two weeks post-GMTH transduction. ${ }^{*} \mathrm{P}<0.05$, as indicated. (B) Flow cytometric analysis of $\alpha$-MHC expression in TTFs post-GMTH induction combined with CIM treatment. (C) Time course of $\alpha$-MHC gene activation in response to GMTH induction and CIM treatment. $\alpha$-MHC, $\alpha$-myosin heavy chain; CIM, cardiac inductive medium; GMTH, ATA binding protein 4, myocyte-specific enhancer factor $2 \mathrm{C}$, T-box transcription factor 5 and heart- and neural crest derivatives-expressed protein 2; TTFs, tail-tip fibroblasts.
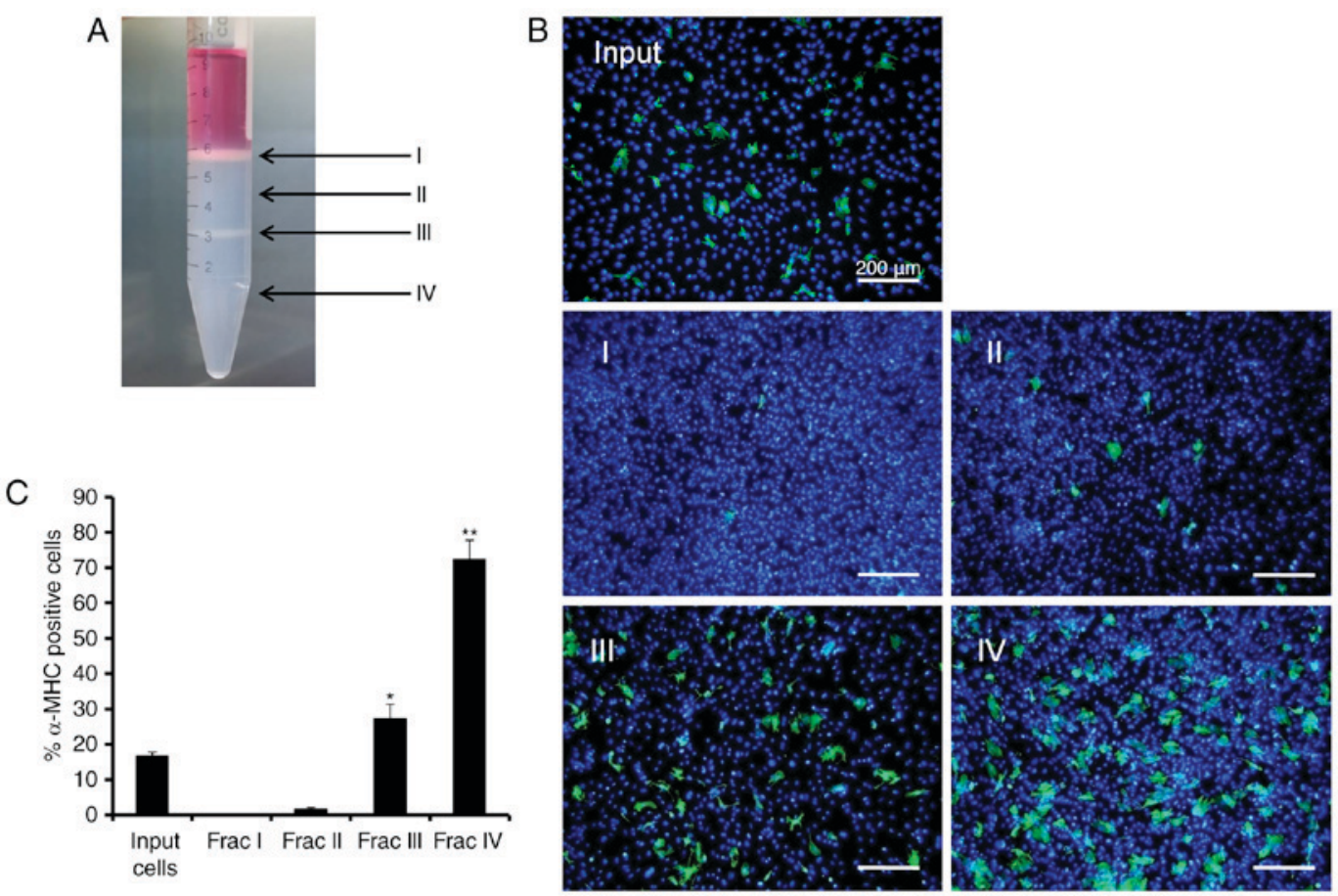

Figure 4. Enrichment of induced cardiomyocytes. (A) The four fractions of induced cardiomyocytes produced following discontinuous Percoll gradient separation. (B) Immunocytochemistry of induced cardiomyocytes following Percoll separation for $\alpha$-MHC(green) and DAPI (blue). Scale bars, $200 \mu \mathrm{m}$. (C) Quantitative analysis of induced cardiomyocytes following Percoll separation. * $\mathrm{P}<0.05$ and ${ }^{* *} \mathrm{P}<0.01$ vs. input cells. $\alpha$-MHC, $\alpha$-myosin heavy chain; Frac, fraction.

Enrichment of induced cardiomyocytes using discontinuous Percoll gradients. CIM-treated TTFs at day 9 post-GMTH transduction were collected and transferred to a discontinuous Percoll gradient (40.5 over 58.5\%). Two layers of cells were generated following centrifugation (Fig. 4A). One layer was observed above the layer of Percoll (fraction I), and the second layer observed was between the two layers of Percoll (fraction III). The input cells, and cells within the $40.5 \%$ Percoll layer (fraction II) and the 58.5\% Percoll layer (fraction IV) were collected for $\alpha$-MHC immunocytochemical staining (Fig. 4B). Quantitative detection of triplicate samples revealed that fraction III contained $27.2 \pm 4.1 \% \alpha-\mathrm{MHC}^{+}$cells and fraction IV contained $72.4 \pm 5.5 \% \alpha-\mathrm{MHC}^{+}$cells, while fraction I and II contained only 0.1 to $1.5 \% \alpha-\mathrm{MHC}^{+}$cells. When 

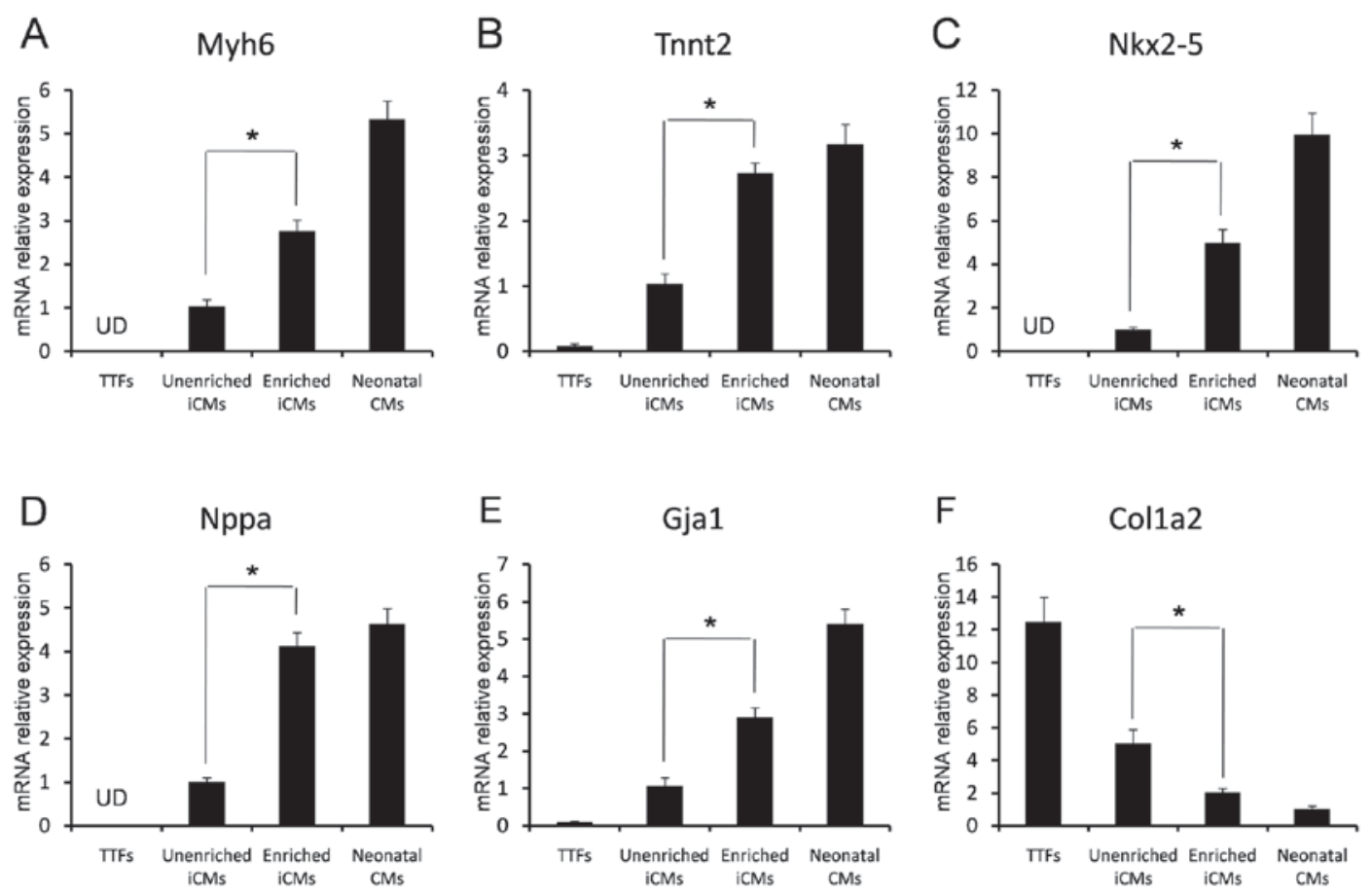

Figure 5. Analysis of cardiac and fibroblast gene expression by reverse transcription-qPCR. The (A) Myh6, (B) Tnnt2, (C) Nkx2-5, (D) Nppa, (E) Gja1 and (F) Colla2 relative mRNA expression levels of TTFs, transduced and unenriched cells, enriched cells from fractions III and IV, and neonatal mouse cardiomyocytes, were determined by qPCR $(\mathrm{n}=3)$. " $\mathrm{P}<0.05$. TTFs, tail-tip fibroblasts; iCMs, induced cardiomyocytes; Myh6, myosin heavy chain 6; Tnnt2, troponin 2; Nkx2-5, NK2 homeobox 5; Nppa, natriuretic peptide A; Gja1, gap junction $\alpha 1$; Col1a2, collagen type 1 a2; qPCR, quantitative polymerase chain reaction.

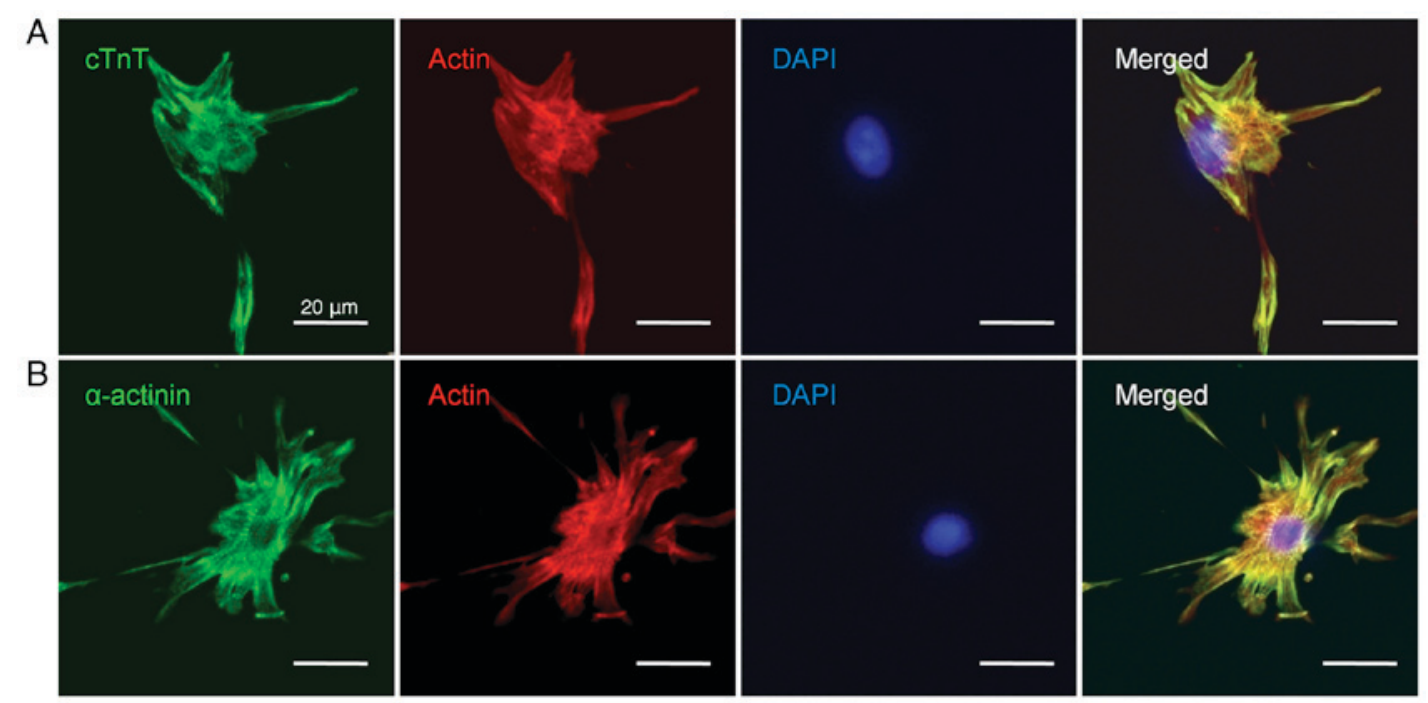

Figure 6. Confocal laser scanning microscopy observation of Percoll-enriched induced cardiomyocytes. Well-developed myofibrillar structures were detected using (A) cTnT and (B) $\alpha$-actin in immunocytochemistry (green). Actin filaments were labeled with phalloidin-tetramethylrhodamine (red). DAPI was used for staining nuclei (blue). Scale bars $=20 \mu \mathrm{m}$. cTnT, cardiac troponin $\mathrm{T}$.

compared with the starting input that constituted $14.7 \pm 1.1 \%$ $\alpha-\mathrm{MHC}^{+}$cells, fraction IV revealed a 3.6-fold enrichment of $\alpha-\mathrm{MHC}^{+}$cells (Fig. 4C). These results demonstrated a marked enrichment of cardiomyocytes using the method of discontinuous Percoll gradient centrifugation.

qPCR analysis of Percoll-enriched cells. The GMTH-transduced cells were collected from fractions III and IV following Percoll separation, and the cardiac gene expressions were compared among TTFs, transduced and unenriched cells, transduced and enriched cells, and neonatal mouse cardiomyocytes. qPCR analysis of gene expression patterns revealed that several cardiac-specific genes were upregulated; however, the fibroblast marker gene, collagen type 1 a2 (COL1A2), was inhibited in Percoll-enriched, GMTH transduced cells (Fig. 5F). Higher expressions of cardiac genes myosin heavy chain 6 (MYH6), troponin 2 (TNNT2), $N K X 2-5$, natriuretic peptide A (NPPA), and gap junction $\alpha 1$ (GJAl) were observed in the enriched cells when compared with transduced and unenriched cells; however, they were not 
detected in TTFs by qPCR (Fig. 5A-E). These data indicated that iCMs could be enriched from GMTH-transduced TTFs. Enriched iCMs had an expression pattern similar to neonatal ventricular cardiomyocytes, and full maturation may be a slow course that occurred over a few of weeks.

Immunocytochemical analysis of Percoll-enriched cells. To characterize the Percoll-enriched cell populations, immunocytochemistry was performed using antibodies against cTnT and $\alpha$-actinin. Confocal laser scanning microscopy revealed that Percoll-enriched cells had strong immunofluorescence for the sarcomeric proteins cTnT and $\alpha$-actinin, and it confirmed the extent of differentiation. The enriched cells exhibited typical cardiomyocyte morphology and possessed distinct myofilaments. The well-developed sarcomeric structures were observed by cTnT and $\alpha$-actinin immunocytochemistry (Fig. 6). Noncardiac cells expressed SMA or vimentin, which was indicative of the existence of fibroblasts or smooth muscle cells in the Percoll-enriched populations (data not shown).

\section{Discussion}

Direct cardiomyocyte reprogramming via the transduction of cardiac transcription factors may be considered as a potential strategy for cardiac regeneration (22). The present study reported that GMTH may facilitate the conversion of mouse TTFs to cardiomyocytes in vitro. In addition, the enrichment of iCMs via Percoll density centrifugation produced a population containing $\sim 72 \% \alpha-\mathrm{MHC}^{+}$cells; enriched iCMs exhibited cardiomyocyte marker expression profiles similar to natural cardiomyocytes, with developed sarcomeric structures. The findings of the present study may provide a novel cell progenitor valuable to cardiovascular disease research and clinical treatment, with minimized risks of immune rejection and tumor formation.

A previous study reported that GMT-mediated induction may reprogram murine cardiac fibroblasts and TTFs into cardiomyocyte-like cells in vitro (9). However, the results of the present study demonstrated that GMT-mediated induction within TTFs induced the expression of a subset of cardiac genes without inducing the cardiac-specific gene, $\alpha$-MHC, supporting previous evidence (17). The addition of HAND2 to GMT induced the expression of $\alpha$-MHC and other cardiac-specific genes the effect of HAND2 on direct cardiomyocyte reprogramming was investigated previously; ectopic expression of HAND2 was demonstrated to enhance the efficiency of reprogramming mammalian fibroblasts to express a cardiac-like profile $(16,23)$. GMTH has been reported to serve as crucial cardiac transcription factors during the early stages of heart development $(24,25)$. GATA4 has been identified as a 'pioneer' factor, which may open chromatin structure in cardiac loci (26); GATA4 facilitates the binding of Mef2c, Tbx 5 and Hand 2 to corresponding binding sites, which may result in the complete activation of cardiac reprogramming. In addition, HAND2, a basic helix-loop-helix transcription factor, has been considered to be an important mediator of cardiomyocyte generation. Within mice, the loss of HAND2 function has been associated with hypoplasia of the right ventricle and outflow tract (27), indicating that cardiomyocyte development may be derived from the second heart field (SHF) and may be enhanced by HAND2. In addition, the deletion of HAND2 function from the SHF via tissue-specific deletion of a conditional allele may influence the survival of this progenitor population (28). HAND2 exhibitsimportant and instructive effects on promoting cardiomyocyte generation by interfering with specification and proliferation processes (29-31).

The results of the present study indicated that cardiomyocyte reprogramming may be markedly induced by CIM treatment, which contains particular cytokines and small molecules that may induce cardiogenesis by modulating the activities of the bone morphogenetic protein (BMP), transforming growth factor $\beta$ (TGF $\beta$ ), wingless-related integration site (Wnt), Notchor Hedgehog signaling pathways (32).

In the present study, CIM treatment was associated with the induction of cardiomyocyte reprogramming. The presence of particular cytokines and small molecules within CIM may modulate the activities of the BMP, TGF $\beta$, Wnt, Notchor Hedgehog pathways (32). Reprogramming factors may induce the progenitor cell population, which may result in a variety of cell types as these progenitor cells regress into epigenetically-stable stages (33-35). Fully differentiated cell types may be obtained via the overexpression of a variety of lineage-specific transcription factors. The effects of BMP4 on direct cardiac differentiation is yet to be investigated; however, BMP4 may serve an important role in the induction of cardiogenesis from nascent precursors during a key developmental stage (36,37).

Following optimization of the reprogramming conditions and determination of the enrichment time, iCMs were predominantly identified in fractions III and IV using a discontinuous Percoll gradient similar to the separation of ESC-derived and natural ventricular cardiomyocytes as observed in previous studies $(38,39)$. qPCR analysis revealed that increased expression levels of cardiac-specific genes, including $M Y H 6$, TNNT2, NKX2-5, NPPA and GJA1 were detected within Percoll-enriched iCMs. In addition, the expression levels of the fibroblast marker gene COL1A2 was downregulated. Confocal laser scanning microscopy analysis revealed that the enriched iCMs exhibited typical cardiomyocyte morphology and formed characteristic sarcomeric structures. Additionally, Percoll-enriched iCMs exhibited an expression profile similar to that of neonatal mouse ventricular cardiomyocytes (9).

The present study demonstrated that a cardiomyocyte-enriched population may be obtained from TTFs transduced with a combination of cardiac-specific transcription factors (GMTH), and enriched iCMs may be applied to cell transplantation, cardiac tissue engineering and drug discovery, amongst other developments. However, numerous challenges remain in transcription factor-based cardiac reprogramming; future investigations may focus on the cardiac reprogramming of human autologous cell sources and transplantation into the infarcted heart for cardiac repair.

\section{Acknowledgements}

The present study was supported in part by the National Key R\&D Program of China (grant no. 2017YFC1104701), the National Basic Science and Development Program (973 Program; grant nos. 2012CB518103, 2012CB518105 and 2013CB127304), the 863 Projects of Ministry of Science and Technology of China (grant nos. 2013AA020105 and 
2012AA020502), the National Natural Science Foundation of China (grant nos. 81121004, 81230041, 31100705, 30901564 and 81101883) and the Beijing Novel Program (grant nos. 2008B53 and 2009A038), and the China Postdoctoral Science Foundation (grant nos. 2013M542517 and 2015T81099).

\section{References}

1. Malliaras K and Marbán E: Cardiac cell therapy: Where we've been, where we are, and where we should be headed. Br Med Bull 98: 161-185, 2011.

2. Zimmermann WH: Remuscularizing failing hearts with tissue engineered myocardium. Antioxid Redox Signal 11: 2011-2023, 2009.

3. Laflamme MA and Murry CE: Regenerating the heart. Nat Biotechnol 23: 845-856, 2005.

4. Zimmermann WH and Eschenhagen T: Embryonic stem cells for cardiac muscle engineering. Trends Cardiovasc Med 17: 134-140, 2007.

5. He W, Ye L, Li S, Liu H, Wu B, Wang Q, Fu X, Han W and Chen Z: Construction of vascularized cardiac tissue from genetically modified mouse embryonic stem cells. J Heart Lung Transplant 31: 204-212, 2012.

6. Takahashi K, Tanabe K, Ohnuki M, Narita M, Ichisaka T, Tomoda K and Yamanaka S: Induction of pluripotent stem cells from adult human fibroblasts by defined factors. Cell 131: 861-872, 2007.

7. Takahashi K and Yamanaka S: Induction of pluripotent stem cells from mouse embryonic and adult fibroblast cultures by defined factors. Cell 126: 663-676, 2006.

8. Zhou Q, Brown J, Kanarek A, Rajagopal J and Melton DA: In vivo reprogramming of adult pancreatic exocrine cells to beta-cells. Nature 455: 627-632, 2008.

9. Ieda M, Fu JD, Delgado-Olguin P, Vedantham V, Hayashi Y, Bruneau BG and Srivastava D: Direct reprogramming of fibroblasts into functional cardiomyocytes by defined factors. Cell 142: 375-386, 2010.

10. Vierbuchen T, Ostermeier A, Pang ZP, Kokubu Y, Südhof TC and Wernig M: Direct conversion of fibroblasts to functional neurons by defined factors. Nature 463: 1035-1041, 2010.

11. Sekiya $S$ and Suzuki A: Direct conversion of mouse fibroblasts to hepatocyte-like cells by defined factors. Nature 475: 390-393, 2011.

12. Szabo E, Rampalli S, Risueño RM, Schnerch A, Mitchell R, Fiebig-Comyn A, Levadoux-Martin M and Bhatia M: Direct conversion of human fibroblasts to multilineage blood progenitors. Nature 468: 521-526, 2010.

13. Takeuchi JK and Bruneau BG: Directed transdifferentiation of mouse mesoderm to heart tissue by defined factors. Nature 459: 708-711, 2009

14. Jayawardena TM, Egemnazarov B, Finch EA, Zhang L, Payne JA, Pandya K, Zhang Z, Rosenberg P, Mirotsou M and Dzau VJ: MicroRNA-mediated in vitro and in vivo direct reprogramming of cardiac fibroblasts to cardiomyocytes. Circ Res 110 1465-1473, 2012

15. Qian L, Huang Y, Spencer CI, Foley A, Vedantham V, Liu L, Conway SJ, Fu JD and Srivastava D: In vivo reprogramming of murine cardiac fibroblasts into induced cardiomyocytes. Nature 485: 593-598, 2012.

16. Song K, Nam YJ, Luo X, Qi X, Tan W, Huang GN, Acharya A, Smith CL, Tallquist MD, Neilson EG, et al: Heart repair by reprogramming non-myocytes with cardiac transcription factors. Nature 485: 599-604, 2012.

17. Chen JX, Krane M, Deutsch MA, Wang L, Rav-Acha M, Gregoire S, Engels MC, Rajarajan K, Karra R, Abel ED, et al: Inefficient reprogramming of fibroblasts into cardiomyocytes using Gata4, Mef2c, and Tbx5. Circ Res 111: 50-55, 2012.

18. Chien KR, Yi BA, Xu H and Mummery CL: Cardiomyocyte reprogramming and the new age of cellular alchemy. J Mol Cell Cardiol 53: 311-313, 2012.
19. Braam SR, Passier R and Mummery CL: Cardiomyocytes from human pluripotent stem cells in regenerative medicine and drug discovery. Trends Pharmacol Sci 30: 536-545, 2009.

20. Livak KJ and Schmittgen TD: Analysis of relative gene expression data using real-time quantitative PCR and the 2(-Delta Delta C(T)) method. Methods 25: 402-408, 2001.

21. He W, Ye L, Li S, Liu H, Wang Q, Fu X, Han W and Chen Z: Stirred suspension culture improves embryoid body formation and cardiogenic differentiation of genetically modified embryonic stem cells. Biol Pharm Bull 35: 308-316, 2012.

22. Doppler SA, Deutsch MA, Lange R and Krane M: Direct reprogramming-the future of cardiac regeneration? Int J Mol Sci 16: 17368-17393, 2015.

23. Nam YJ, Song K, Luo X, Daniel E, Lambeth K, West K, Hill JA, DiMaio JM, Baker LA, Bassel-Duby R and Olson EN: Reprogramming of human fibroblasts toward a cardiac fate. Proc Natl AcadSci USA 110: 5588-5593, 2013.

24. Olson EN: Gene regulatory networks in the evolution and development of the heart. Science 313: 1922-1927, 2006.

25. Srivastava D: Making or breaking the heart: From lineage determination to morphogenesis. Cell 126: 1037-1048, 2006.

26. Cirillo LA, Lin FR, Cuesta I, Friedman D, Jarnik M and Zaret KS: Opening of compacted chromatin by early developmental transcription factors HNF3 (FoxA) and GATA-4. Mol Cell 9: 279-289, 2002.

27. Srivastava D, Thomas T, Lin Q, Kirby ML, Brown D and Olson EN: Regulation of cardiac mesodermal and neural crest development by the bHLH transcription factor, dHAND. Nat Genet 16: 154-160, 1997.

28. Tsuchihashi T, Maeda J, Shin CH, Ivey KN, Black BL, Olson EN, Yamagishi $\mathrm{H}$ and Srivastava D: Hand2 function in second heart field progenitors is essential for cardiogenesis. Dev Biol 351: 62-69, 2011.

29. Yelon D, Ticho B, Halpern ME, Ruvinsky I, Ho RK, Silver LM and Stainier DY: The bHLH transcription factor hand2 plays parallel roles in zebrafish heart and pectoral fin development. Development 127: 2573-2582, 2000.

30. Schoenebeck JJ, Keegan BR and Yelon D: Vessel and blood specification override cardiac potential in anterior mesoderm. Dev Cell 13: 254-267, 2007.

31. Schindler YL, Garske KM, Wang J, Firulli BA, Firulli AB, Poss KD and Yelon D: Hand2 elevates cardiomyocyte production during zebrafish heart development and regeneration. Development 141: 3112-3122, 2014.

32. Efe JA, Hilcove S, Kim J, Zhou H, Ouyang K, Wang G, Chen J and Ding S: Conversion of mouse fibroblasts into cardiomyocytes using a direct reprogramming strategy. Nat Cell Biol 13: 215-222, 2011 .

33. Meshorer E, Yellajoshula D, George E, Scambler PJ, Brown DT and Misteli T: Hyperdynamic plasticity of chromatin proteins in pluripotent embryonic stem cells. Dev Cell 10: 105-116, 2006.

34. Hochedlinger $\mathrm{K}$ and Plath K: Epigenetic reprogramming and induced pluripotency. Development 136: 509-523, 2009.

35. Artyomov MN, Meissner A and Chakraborty AK: A model for genetic and epigenetic regulatory networks identifies rare pathways for transcription factor induced pluripotency. PLoS Comput Biol 6: e1000785, 2010.

36. Cohen ED, Tian Y and Morrisey EE: Wnt signaling: An essential regulator of cardiovascular differentiation, morphogenesis and progenitor self-renewal. Development 135: 789-798, 2008.

37. Klaus A and Birchmeier W: Developmental signaling in myocardial progenitor cells: A comprehensive view of Bmp- and Wnt/beta-catenin signaling. PediatrCardiol 30: 609-616, 2009.

38. Xu C, Police S, Rao N and Carpenter MK: Characterization and enrichment of cardiomyocytes derived from human embryonic stem cells. Circ Res 91: 501-508, 2002.

39. E LL, Zhao YS, Guo XM, Wang CY, Jiang H, Li J, Duan CM and Song Y: Enrichment of cardiomyocytes derived from mouse embryonic stem cells. J Heart Lung Transplant 25: 664-674, 2006. 